\title{
Isolation and Application of Mutants Producing Sufficient Isoamyl Acetate, a Sake Flavor Component
}

\author{
Shinzo Ashida, Eiji IchIKawa, Koji Suginami \\ and Satoshi IMAYASU \\ Gekkeikan Sake Company, Ltd., Research Laboratory, 25 Shimotoba \\ Koyanagi-cho, Fushimi-ku, Kyoto 612, Japan \\ Received January 12, 1987
}

\begin{abstract}
Mutants of Saccharomyces cerevisiae that produce sufficient isoamyl acetate were isolated from sake yeast. 5,5,5-Trifluoro-DL-leucine, an analogue of L-leucine, was used for the isolation to eliminate the feedback inhibition by accumulated L-leucine. The concentration of isoamyl alcohol increased about three or four times with these mutants and a sufficient concentration of isoamyl acetate, one of the key components of the sake flavor, was obtained consequently. Mutants producing sufficient isoamyl acetate were also isolated from wine, shochu and beer yeasts by the same method.
\end{abstract}

Isoamyl acetate is one of the most important components of the sake flavor. Recently, many reports ${ }^{1 \sim 5)}$ have appeared on the biosynthesis of isoamyl acetate from isoamyl alcohol through the catalysis of alcohol acetyltransferase (AATase). We reported ${ }^{1)}$ that the production of isoamyl acetate depended highly on the concentration of isoamyl alcohol and the activity of AATase, because enough acetyl-CoA, another source for isoamyl acetate production, existed in the Saccharomyces cerevisiae cells.

AATase is unstable as to high temperature, ${ }^{1)}$ therefore, low temperature fermentation is required to increase the isoamyl acetate concentration when brewing ginjoshu, a special grade of sake with an excellent flavor. Yoshizawa et $a l .{ }^{6}{ }^{1}$ proposed lipase treatment of rice, which enhances the AATase activity by removing unsaturated fatty acids, before fermentation. This is based on the fact ${ }^{5}$ that unsaturated fatty acids inhibit AATase in the cell membrane. We also reported ${ }^{7)}$ that the addition of glucoamylase during the fermentation process increased the concentration of isoamyl acetate. But in none of these cases was the isoamyl acetate concentration obtained sufficient for a flavor component.
There are two major routes for the biosynthesis of isoamyl alcohol. One is via $\alpha$-keto isocaproate in the pathway of L-leucine synthesis from glucose (Fig. 1). The other is the so called Ehrlich mechanism ${ }^{8}$ from L-leucine in rice and koji through transamination. In sake fermentation, a decrease in isoamyl alcohol production with the L-leucine synthesis pathway is caused by inhibition by accumulated Lleucine. Because the activity of $\alpha$-isopropylmalate synthase $^{9)}(\alpha$-IPM synthase), among the enzymes used for L-leucine synthesis, is feedback inhibited or repressed by the accumulated L-leucine. From these facts, it is supposed that mutants released from inhibition of $\alpha$-IPM synthase by L-leucine would produce sufficient isoamyl alcohol.

Ylänen ${ }^{10)}$ selected mutants that produced sufficient isoamyl alcohol using norvaline, an analogue of L-leucine. But they did not mention the formation of isoamyl acetate and did not explain the mechanism for getting rid of the feedback inhibition and the repression of $\alpha$-IPM synthase by accumulated L-leucine. Ouchi et al. ${ }^{11)}$ reported that they selected mutants by the same procedure from Saccharomyces cerevisiae RIB 6006 using norvaline and norleucine, but they could not ob- 


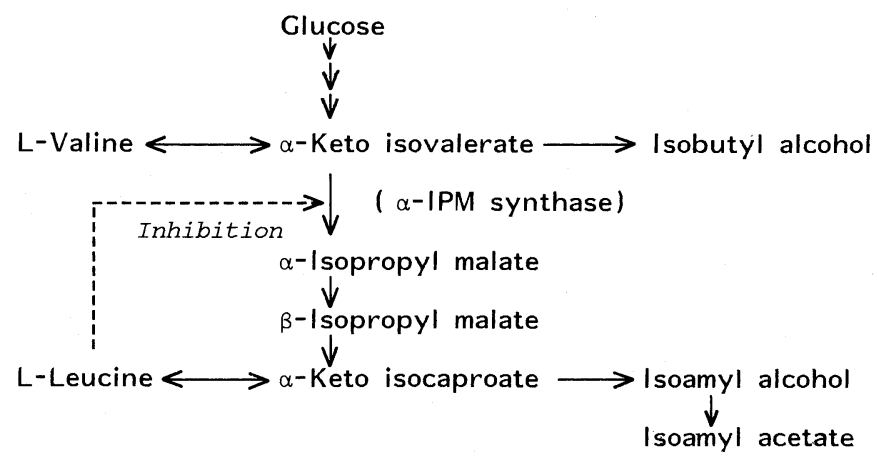

Fig. 1. Pathways for Isoamyl Alcohol Production.

tain mutants producing sufficient isoamyl acetate.

In this paper, we describe a procedure for obtaining mutants free from the feedback inhibition by accumulated L-leucine and the results of a fermentation test with the mutants obtained.

\section{MATERIALS AND METHODS}

Strains. Saccharomyces cerevisiae RIB 6002 and its haploids, G1101(a), G1103( $\alpha$ ) and RIB 6006, were used as sake yeasts. Saccharomyces cerevisiae OC-2, Saccharomyces cerevisiae Kyokaishochu-2 and Saccharomyces uvarum IFO 0565 were also used as wine, shochu and beer yeasts, respectively.

Isolation of mutants resistant to the analogue. The cells were suspended in $5 \mathrm{ml}$ of $0.2 \mathrm{M}$ phosphate buffer, $\mathrm{pH} 8.0$, containing $2 \%$ glucose, and then $0.25 \mathrm{ml}$ of ethyl methanesulfonate (EMS) was added to this suspension. After 60 minutes gentle shaking at $30^{\circ} \mathrm{C}, 0.5 \mathrm{ml}$ of the suspension was spread on a YNB agar plate $(0.67 \%$ Difco yeast nitrogen base, $2 \%$ glucose, $2 \%$ agar) containing $1 \mathrm{~mm}$ 5,5,5-trifluoro-DL-leucine. The colonies that developed on the agar plate were isolated as mutants resistant to the analogue of L-leucine.

Screening of mutants producing sufficient isoamyl acetate. All the resistant mutants were cultured in the YM liquid medium $(5 \%$ glucose, $0.5 \%$ polypeptone, $0.3 \%$ yeast extract, $0.3 \%$ malt extract) for two days at $30^{\circ} \mathrm{C}$. The flavor components of the broth were measured by head space gas chromatography.

Fermentation test. In the case of sake, an ordinary grade of rice (Nihonbare) was used with a polishing ratio of $72 \%$, and the sake mash was composed of $1040 \mathrm{~g}$ of steamed rice, $240 \mathrm{~g}$ of rice koji and $1400 \mathrm{ml}$ of water. The yeast cultured in $\mathrm{YM}$ liquid medium was added at the concentration of $1.4 \times 10^{7}$ cells $/ \mathrm{ml}$ and then the mash was fermented at $15^{\circ} \mathrm{C}$.

In the case of wine, Koshu grapes were used. $1430 \mathrm{~g}$ of grapes were crushed and pressed, and one liter of juice was obtained. The sugar concentration of the must was brought to $24 \%$ with glucose. The yeast was added at the concentration of $2 \times 10^{7}$ cells $/ \mathrm{ml}$, and the must was fermented at $20^{\circ} \mathrm{C}$.

Growth of the organism and preparation of a crude extract. The cells used for the enzyme preparation were grown on YNB medium containing $2 \mathrm{mM}$ L-leucine to obtain a higher level of $\alpha$-IPM synthase activity. ${ }^{9)}$ A crude extract of the cells was prepared by the method of Ulm et $a .^{12)}$

Enzyme assay. The activity of $\alpha$-IPM synthase was measured by the method of Ulm et al., ${ }^{12)}$ determining the amount of $\mathrm{COA}$ liberated in a certain period of time with 5,5-dithiobis-(2-nitrobenzoate)

Investigation of feedback inhibition and repression of $\alpha$ IPM synthase by L-leucine. The feedback inhibition was investigated as the sensitivity to $5 \mathrm{mM} \mathrm{L}$-leucine of $\alpha$-IPM synthase in the crude extracts. The repression was examined in comparison with the $\alpha$-IPM synthase activity in a crude extract from the yeast cells cultured on YNB liquid medium with $5 \mathrm{~mm}$ L-leucine and L-threonine.

\section{RESULTS}

\section{Selection of an analogue}

To select an appropriate analogue of Lleucine, the effects of analogues on the growth of Saccharomyces cerevisiae G1103 were examined. $1 \mathrm{~mm}$ 5,5,5-Trifluoro-DL-leucine in the YNB medium completely inhibited the growth of the yeast, while 4-aza-DL-leucine, 2-thiazolyl-DL-alanine, DL-norvaline, DL-norleucine 


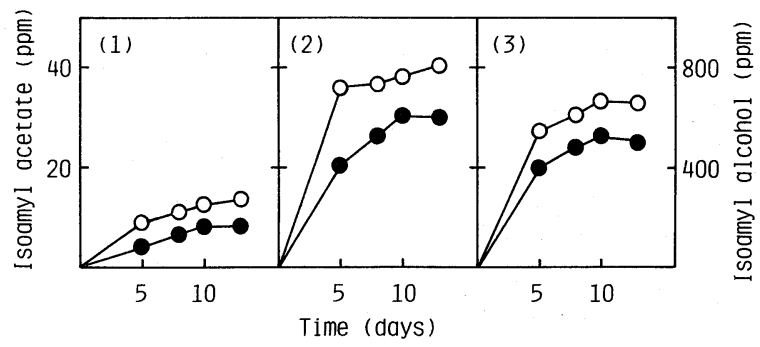

FIG. 2. Changes in the Concentrations of Isoamyl Alcohol and Isoamyl Acetate on Sake Fermentation. (1), wild; (2), F-3; (3), F-7. (○), isoamyl acetate; (O), isoamyl alcohol.

and DL-2- $\alpha$-aminobutyric acid showed no inhibition, even at the concentration of $10 \mathrm{~mm}$. On the basis of the results, 5,5,5-trifluoro-DLleucine was used for the isolation hereafter.

\section{Isolation and screening of mutants producing sufficient isoamyl acetate}

Mutants were induced from Saccharomyces cerevisiae G1103 by EMS treatment and used for the isolation in the medium containing 5,5,5-trifluoro-DL-leucine. Thirty five colonies were isolated as analogue resistant strains. These strains were subjected to the following screening. Table I shows the results of the first screening. All the selected strains produced much higher concentrations of isoamyl alcohol and isoamyl acetate than the wild strain. These mutants are supposed to be free from inhibition of $\alpha$-IPM synthase by accumulated Lleucine. F-3 and F-7 were used for the following fermentation test since they produced the highest concentrations of isoamyl acetate among the mutants obtained.

\section{Sake fermentation test}

Smooth fermentations were performed using the wild, F-3 and F-7 strains. Figure 2 shows the changes in the concentrations of isoamyl alcohol and isoamyl acetate on sake fermentation. Sufficient isoamyl alcohol and isoamyl acetate were produced by both the F-3 and F-7 strains. The isoamyl acetate content had already reached $20 \mathrm{ppm}$ at 5 days. Table II shows the results of analysis of crude sake. The concentration of alcohol was not so high because the amount of water added initially was
Table I. The First Screening for Mutants Producing Sufficient Isoamyl Acetate

\begin{tabular}{lcccc}
\hline & $\begin{array}{c}n \text {-Propyl } \\
\text { alcohol } \\
(\mathrm{ppm})\end{array}$ & $\begin{array}{c}\text { Isobutyl } \\
\text { alcohol } \\
(\mathrm{ppm})\end{array}$ & $\begin{array}{c}\text { Isoamyl } \\
\text { alcohol } \\
(\mathrm{ppm})\end{array}$ & $\begin{array}{c}\text { Isoamyl } \\
\text { acetate } \\
(\mathrm{ppm})\end{array}$ \\
\hline Wild & 10.0 & 16.6 & 65.7 & 0.38 \\
F-1 & 9.6 & 13.0 & 190.2 & 0.88 \\
F-3 & 9.7 & 15.3 & 204.2 & 1.00 \\
F-7 & 9.8 & 11.8 & 220.1 & 1.64 \\
F-13 & 9.2 & 11.2 & 201.0 & 1.79 \\
F-35 & 9.2 & 12.1 & 199.3 & 0.75 \\
\hline
\end{tabular}

Table II. Analysis of Crude Sake

\begin{tabular}{lrrr}
\hline Component & Wild & F-3 & F-7 \\
\hline Sake meter & +5.1 & -2.8 & +3.8 \\
Alcohol $(\%)$ & 16.6 & 16.2 & 16.2 \\
Total acid $(\mathrm{ml})$ & 2.6 & 2.6 & 2.4 \\
Amino acid $(\mathrm{ml})$ & 1.7 & 1.9 & 1.6 \\
\hline & & & \\
& & & \\
& & & \\
n-Propyl alcohol & 85.3 & 78.0 & 66.3 \\
Isobutyl alcohol & 125.6 & 38.9 & 47.1 \\
Isoamyl alcohol & 272.6 & 810.7 & 666.4 \\
Isoamyl acetate & 7.7 & 29.6 & 25.3 \\
Ethyl caproate & 1.8 & 2.2 & 1.5 \\
\hline
\end{tabular}

$140 \%$ over rice. There were little differences in total acids and amino acids among the three samples. The concentrations of isoamyl alcohol and isoamyl acetate with F-3 and F-7 became three or four times as high as those with the wild strain. In an organoleptic test, the crude sake with F-3 and F7 showed an excellent fruity flavor. A high content of isoamyl alcohol had little effect on the sake flavor because the flavor of isoamyl acetate was 


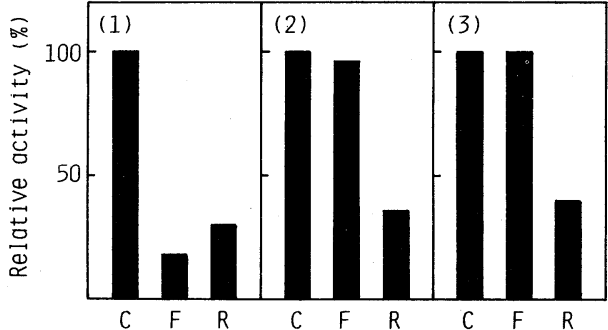

FIG. 3. Effect of L-Leucine as to Feedback Inhibition or Repression of $\alpha$-IPM Synthase Activity.

(1), wild; (2), F-3; (3), F-7.

$\mathrm{C}$, control; F, feedback inhibition; $\mathrm{R}$, repression. The $\cdot \alpha-$ IPM synthase activity in a control is taken as $100 \%$.

much stronger than that of isoamyl alcohol in sake obtained with F-3 and F-7. The concentration of isobutyl alcohol decreased by one-third with F-3 and F-7, but the other components, such as $n$-propyl alcohol, were almost the same among the three samples.

\section{Investigation of the release from inhibition}

The addition of $5 \mathrm{~mm}$ L-leucine completely inhibited $\alpha$-IPM synthase activity in the wild strain. On the other hand, the activity of $\alpha$ IPM synthase in F-3 and F-7 was not inhibited by the addition of $5 \mathrm{~mm}$ L-leucine (Fig. 3). When the cells were grown with $5 \mathrm{~mm}$ L-leucine and L-threonine, the activity of $\alpha$-IPM synthase in the three strains was repressed by $60 \sim 70 \%$. These results indicate that the release from inhibition is not caused by the repression but by the feedback inhibition by Lleucine.

\section{Mating experiment}

The mutant strains, F-3 and F-7, were assumed to be dominant in diploids because they contained $\alpha$-IPM synthase insensitive to L-leucine. We performed mating experiments to confirm the dominance of these mutants. Figure 4 shows the mating experiment scheme. F-3-3( $\alpha$, met), F-7-22( $\alpha$, met) and G1503(a, his) were isolated from F-3, F-7 and G1101, respectively. Then, M-3 and M-7 were obtained as shown in the scheme. The M-3 and M-7 strains produced almost equal amounts of isoamyl alcohol to F-3 and F-7 in the fremen-

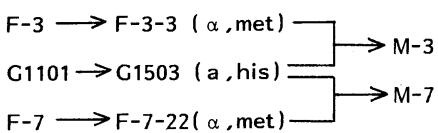

FIG. 4. Mating Experiment.

Table III. Isoamyl Alcohol Synthesis in CRude SaKe

\begin{tabular}{lc}
\hline Strains & Isoamyl alcohol (ppm) \\
\hline G1101 & 254 \\
F-3 & 835 \\
F-7 & 799 \\
M-3 & 646 \\
M-7 & 799 \\
\hline
\end{tabular}

Table IV. Fermentation Test on Diploids

\begin{tabular}{llccc}
\hline Strains & & $\begin{array}{c}\text { Alcohol } \\
(\%)\end{array}$ & $\begin{array}{c}\text { Isoamyl } \\
\text { alcohol } \\
(\mathrm{ppm})\end{array}$ & $\begin{array}{c}\text { Isoamyl } \\
\text { acetate } \\
(\mathrm{ppm})\end{array}$ \\
\hline G1101 (wild) & Haploid & 17.1 & 256 & 5.4 \\
F-3 & Haploid & 16.5 & 859 & 27.3 \\
RIB6002 & Diploid & 17.3 & 267 & 4.0 \\
6002-F & Diploid & 16.6 & 732 & 11.5 \\
RIB6006 & Diploid & 18.1 & 238 & 4.4 \\
6006-F & Diploid & 18.2 & 778 & 18.8 \\
\hline
\end{tabular}

tation test (Table III). These results indicate that the gene involved in mutants producing sufficient isoamyl alcohol is dominant and that it is possible to also obtain strains producing sufficient isoamyl acetate from the diploid easily. We tried to obtain similar mutants from sake, wine, shochu and beer yeasts as diploids. All these mutants showed high isoamyl alcohol productivity on the YM medium. Table IV shows the results of the fermentation test using the mutants isolated from sake yeast (RIB6002, RIB6006). These superior mutants were stable on transfer for cultivation for more than fifteen times.

\section{Wine fermentation test}

Wine was made using the resistant mutant (OC-2-F) isolated from Saccharomyces cerevisiae OC-2. Table $\mathrm{V}$ shows the results of the fermentation test with the mutant. The con- 
Table V. Fermentation Test on Wine Produced by Mutants

\begin{tabular}{lrc}
\hline & OC-2 & OC-2-F \\
\hline Alcohol $(\%)$ & 12.8 & 12.8 \\
Isoamyl alcohol $(\mathrm{ppm})$ & 384.8 & 568.0 \\
Isoamyl acetate $(\mathrm{ppm})$ & 10.9 & 20.6 \\
\hline
\end{tabular}

centrations of isoamyl alcohol and isoamyl acetate with OC-2-F increased to approximately twice as much as those with OC-2. The wine obtained with OC-2-F also had an excellent fruity flavor. There was little difference in alcohol content between them. We consider that mutants resistant to the analogue of L-leucine can be used not only for sake production but also for the production of other alcoholic beverages such as wine, shochu and beer.

\section{DISCUSSION}

All the mutants resistant to 5,5,5-trifluoroDL-leucine produced sufficient concentrations of isoamyl alcohol and isoamyl acetate. Ouchi et al. ${ }^{11)}$ used norvaline and norleucine as analogues but could not obtain useful mutants. This seems to be caused by the imperfect inhibition by these analogues of the growth of yeasts. We assumed that 5,5,5-trifluoro-DLleucine is one of the most appropriate analogues for isolating desirable mutants.

The concentration of isoamyl alcohol in crude sake produced with F-3 and F-7 had increased approximately four times, and the concentration of isobutyl alcohol decreased by one-third compared with in the wild strain. This indicates that the feedback inhibition of $\alpha$-IPM synthase by accumulated L-leucine is decreased in the mutants, so that more $\alpha$ isopropyl malate can be synthesized instead of isobutyl alcohol from $\alpha$-keto isovalerate.

The increase in isoamyl acetate coincided with the increase in isoamyl alcohol. This supports the hypothesis in our previous paper $^{1)}$ that the production of isoamyl acetate depends highly on the isoamyl alcohol concentration.

The mutation in F-3 and F-7 seems to occur on the structural gene for $\alpha$-IPM synthase and to be dominant. This means that desirable mutants can be obtained directly from the diploids, i.e., the wild strains. In practice a large number of mutants producing sufficient isoamyl acetate could be isolated from diploid strains used for the brewing of various kinds of alcoholic beverages. The use of yeast diploids is of great advantage for practical brewing. In the case of brewers yeasts, since the ability of spore formation is poor, the isolation of their haploid strains is not easy. Even if haploids are obtained, they cannot be applied for practical brewing.

In brewing sake, highly polished rice and low temperature fermentation have been required so previously to obtain a sufficient concentration of isoamyl acetate. By using mutants resistant to 5,5,5-trifluoro-DL-leucine, an excellent sake flavor can be obtained even on ordinary fermentation.

\section{REFERENCES}

1) K. Kuriyama, S. Ashida, Y. Saito, K. Suginami and S. Imayasu, Hakkokogaku, 64, 169 (1986).

2) T. Ishikawa and K. Yoshizawa, Agric. Biol. Chem., 43, 59 (1979).

3) K. Yoshioka and N. Hashimoto, Agric. Biol. Chem., 45, 2183 (1981).

4) T. Ishikawa, H. Momose and K. Yoshizawa, Nippon Jōzōkyōkai Zasshi, 79, 62 (1984).

5) K. Yoshioka and N. Hashimoto, Agric. Biol. Chem., 47, 2287 (1983).

6) K. Yoshizawa, M. Ikemi and T. Ishikawa, Nippon Jōzōkyōkai Zasshi, 71, 975 (1975).

7) K. Suginami, Koji Kenkyu Kaiho, 17, 31 (1981).

8) C. Rainbow, "The Yeast," Vol. III, ed. by J. S. Harrison and A. H. Rose, Academic Press Inc., New York, 1970, pp. 190 199 .

9) T. Satyanarayana, H. E. Umbarger and G. Lindegren, J. Bacteriol., 96, 2018 (1968).

10) L. Ylänen, J. Inst. Brew., 72, 50 (1966).

11) K. Ouchi, K. Sato, N. Miyajima, T. Araki and H. Akiyama, Nippon Jōzōkyōkai Zasshi, 76, 843 (1981).

12) E. H. Ulm, R. Bohme and G. Kohlhaw, J. Bacteriol., 110, 1118 (1972). 